\title{
DOI: 10.36377/1683-2981-2021-19-3-160-165 \\ Comparative Evaluation of effect of resin protective coatings on the surface hardness and shear punch strength of different GICs - An InVitro Study
}

\begin{abstract}
(o Niharika Mishra', Manish Agarwal', MP Singh'3 , Apoorva Khullar', Khushboo Magwa², Sonal Singh Thakur ${ }^{5}$ 'Department of Conservative Dentistry \& Endodontics, Mansarovar Dental College Hospital and Research Centre, Bhopal, India 2Department of Conservative Dentistry \& Endodontics, People's College of Dental Sciences and Research Centre, Bhopal, India ${ }^{3}$ Dentistry Department, Chirayu Medical College and Hospital, Bhopal, India ${ }^{4}$ Department of Conservative Dentistry \& Endodontics, Rishiraj College of Dental Science and Research Centre, Bhopal India ${ }^{5}$ Department of Conservative Dentistry \& Endodontics, Sri Aurobindo college of dentistry, Indore, India
\end{abstract}

\begin{abstract}
:
G- Coat Plus is a nano-filled, self-adhesive light cured protective coating that strengthens, protects and enhances glass ionomer restorations. The effect of G- Coat plus on different GICs have not been studied comprehensively. Hence the study was conducted to compare the effect of resin coating on surface hardness and Shear punch strength of newer modified GIC.

Materials and methods: 120 samples of three GICs i.e. Fuji IX Extra, Fuji IX Fast, Fuji II LC were prepared using specialized metal moulds. Specimens of Fuji II LC were light cured for 20 seconds. Coated and uncoated samples were tested for surface hardness and 30 samples were tested for shear punch strength. $P<0.05$ was considered as significant.

Results: Shear Punch Strength after G-Coat plus coating was significantly higher for Fuji IX Extra followed by Fuji II LC. Surface Hardness after G. Coat plus coating was significantly higher for Fuji IX Extra followed by Fuji IX Fast and Fuji II LC.

Conclusion: Surface coating by a resin agent significantly increases the surface hardness and shear strength of the GIC. Considering the above stated findings, they can be incorporated into daily clinical practice to enhance the strength and longevity of GIC.
\end{abstract}

Keywords: GIC, Shear strength, surface hardness, aesthetic, restorative material.

Received: 24.05.2021; accepted: 29.08.2021.

Conflict of interests: The authors declare no conflict of interests.

For citation: Niharika Mishra, Manish Agarwal, MP Singh, Apoorva Khullar, Khushboo Magwa, Sonal Singh Thakur. Comparative Evaluation of effect of resin protective coatings on the surface hardness and shear punch strength of different GICs - An InVitro Study. Endodontics to day. 2021; 19(3):160-165. DOI: 10.36377/1683-2981-2021-19-3-160-165.

\section{INTRODUCTION}

During the last decades, there has been a surge in the variety of dental restorative materials such as gold, ceramic and amalgam. Their limitations such as brittleness in case of ceramic, toxicity by mercury in amalgam have limited their extensive use. Great strides in research have led to a variety of alternatives with an easy restorative technique compared to amalgam. The most common are resin composites and glass-ionomer cements (GICs) [1].

GIC was invented by Wilson and Kent in 1969 at the Laboratory of the Government Chemist in London, United Kingdom [2]. These materials are used extensively in restorative dentistry largely due to their adhesive, toothcoloured, anti-cariogenic properties and their usefulness in variety of clinical situations as restorative, lining, luting and sealing; no other restorative material has such wide applications [3].

However, poor mechanical properties, such as low fracture strength, toughness and wear, limit their extensive use as a filling material in stress-bearing posterior dental region. The evolution of the GIC over the last decades has resulted in changes in both the glass powder component and liquid, polycarboxylic acid component. Several approaches dealt with incorporation of second phase ceramic or glass fibers or with metal particles. Encouraging results were also obtained by compounding reactive glass fibers [4]. Newer generations of GIC have been developed each overcoming the drawback of the former. The Fuji IX Extra, Fuji IX Fast and Fuji II LC (GC Corporation, Tokyo, Japan).

Hardness and shear punch strength are two tests that can be used to evaluate those mechanical properties [5]. The cements are tremendously susceptible to hydration and dehydration during their setting process, it leads to dimensional alteration, microcracks and lack of adhesion. To decrease the vulnerability of conventional GIC and its hybrid version to moisture, surface protectors are recommended, such products include varnishes, petroleum jelly (solid, liquid), nail varnishes and resin coatings [6].

G- Coat Plus (GC Corporation, Tokyo, Japan) is a nano-filled, self-adhesive light cured protective coating that strengthens, protects and enhances glass ionomer restorations. The effect of G-Coat plus on different GICs have not been studied comprehensively with varying results $[7,8]$. Hence the aim of present study was to compare and evaluate the effect of resin coating on two 


\section{Исследования / Scientific researches 161}

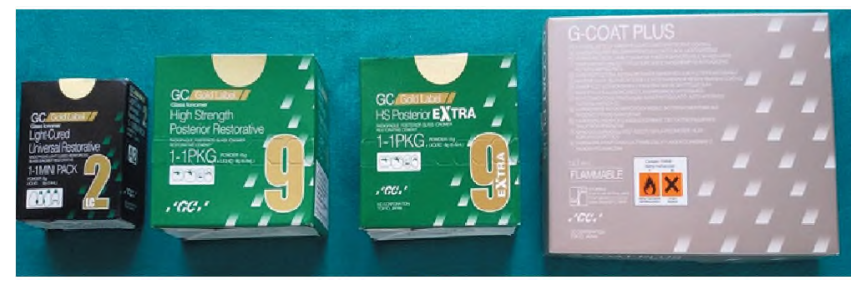

Fig. 1. Armamentarium used in the study - (Fuji II LC, Fuji IX Fast, Fuji IX Extra, G-Coat Plus)

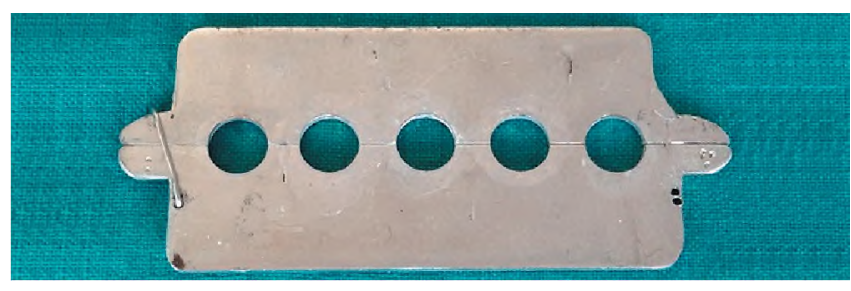

Fig. 2. Stainless steel split moulds (Custom made)

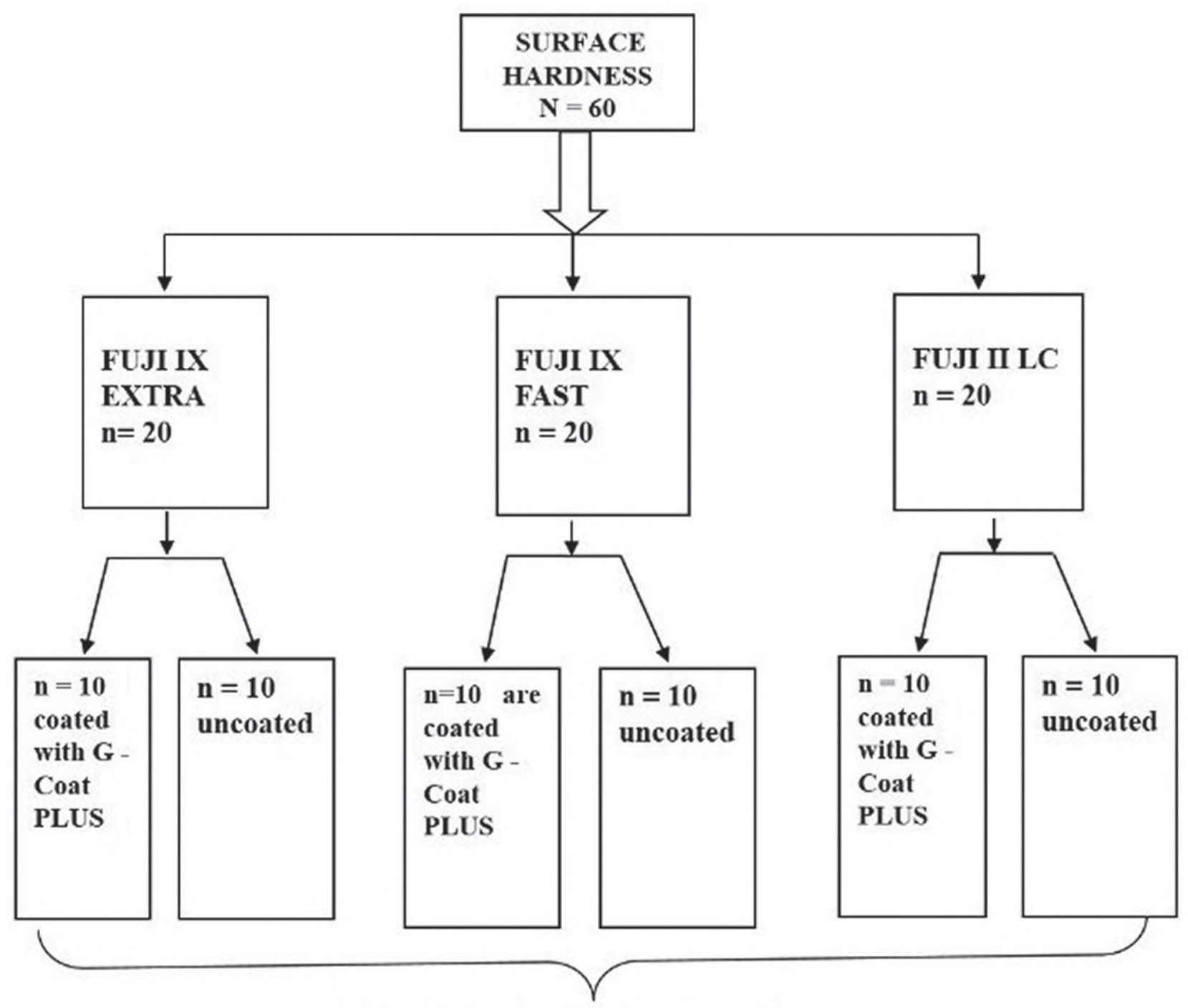

Stored in saline at $37^{\circ} \mathrm{C}$ for two weeks

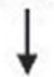

All samples are subjected to Vickers micro hardness tester

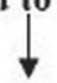

$100 \mathrm{gm}$ load was applied.

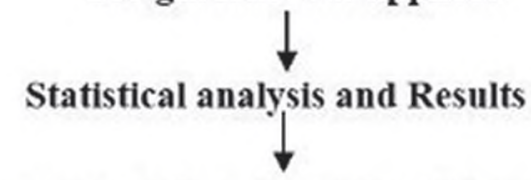

Three readings were taken for each specimen and mean was calculated.

Fig. 4. Schematic illustration showing protocol followed for surface hardness 


\section{$162 \mid$ исследования / Scientific researches}

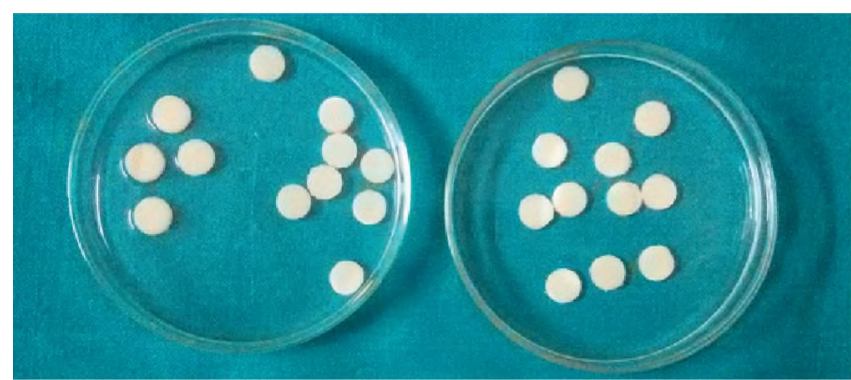

Fig. 3. GIC samples without coat and with coat in saline

important properties-Surface hardness and Shear punch strength of newer modified GICs discussed above.

\section{MATERIALS \& METHODS}

The present study was approved by the institutional ethical board of People's college of dental sciences and research institute [2014CON02]. 120 samples $(N=120)$ three GICs i.e. Fuji IX Extra (GC Corporation), Fuji IX Fast (GC Corporation), Fuji II LC (GC Corporation) (fig. 1) were prepared using customized metal moulds with dimensions (10 $\mathrm{mm} \times 2 \mathrm{~mm}$ ) (fig. 2). These specimens were prepared by compressing the GIC material between two glass slides $(76 \mathrm{~mm} \times 26 \mathrm{~mm} \times 1 \mathrm{~mm})$, separated by the split metal mould. The upper surfaces of unset specimen were covered with thin Mylar strips (Rite Dent, India). Specimens of Fuji II LC were irradiated using a Blue phase CB Polywave LED polymerization equipment at $1200 \mathrm{~mW} / \mathrm{cm}^{2}$ for 20 seconds. The polymerization of the specimen was carried out on each side top and bottom sides against the strip and glass slides and samples were polymerized for 20 seconds after removal from the mould. Then specimens of each group were divided into subgroups of 10 into which 10 were coated with G- Coat Plus resin coating and irradiated using Bluephase $C B$ Polywave LED polymerization equipment at $1200 \mathrm{~m} \mathrm{W/ \textrm {cm } ^ { 2 }}$ for 20 seconds.

Inclusion criteria: Samples free of any defects and scratches when observed under magnification. Exclusion criteria: Samples with any uneven margins, any defects and scratches.

The Surface hardness specimens were removed from the mould and any flash of Material was trimmed away with sand paper. Then all the specimens were kept in Saline at $37^{\circ} \mathrm{C}$ prior to testing for 2 weeks (fig. 3). For Surface Hardness testing, prepared samples were tested and evaluated using the Vicker's microhardness tester (Reichert Austria Make, Sr. No. 363798). The Diamond Indentor is positioned on the sample surface. $100 \mathrm{~g}$ load applied for 20 seconds dwell time. Indentor is removed \& Indentation measured. Vicker Hardenss is noted from the Chart provided. The Shear punch strength specimens were removed from the mould and any flash of Material was trimmed away with sand paper. Then all the specimens were kept in distilled water at $37^{\circ} \mathrm{C}$ prior to testing for 2 weeks. The protocol followed for testing is illustrated in figure 4 and 5 .

\section{Specimen testing for Shear Punch Strength}

All the specimens were kept individually in the micro punch apparatus mounted on Universal testing machine (Star testing systems, India). The thickness of each specimen was measured with the digital micrometer (Mitutoyo, Japan) with an accuracy of $0.001 \mathrm{~mm}$. The compressive force was applied on the punch at cross head speed of $1 \mathrm{~mm} / \mathrm{min} \&$ maximum load was recorded.

Shear punch strength was calculated using formula:

\author{
Shear strength $(\mathrm{MPa})=\frac{\text { Force }(\mathrm{N})}{3.14 \times \text { Punch diameter }(\mathrm{mm}) \times}$ \\ Thickness of Specimen (mm)
}

Data was entered in Microsoft excel. Mean and standard deviation (SD) of shear punch strength (MPa) in different glass ionomer/restorative materials with or without resin coat were calculated. Shapiro-Wilk test showed that shear punch strength (MPa) follow normal distribution. Hence parametric test, two way ANOVA (analysis of variance) followed by LSD post Hoc test was applied for comparison between different groups. $P$ value $<0.05$ was considered statistically significant. All analyses were performed using version 21.0 of the Statistical Package for social sciences (IBM Corporation, Armonk, New York, U.S.A.).

\section{RESULTS}

For Shear Punch Strength testing, the thickness of each specimen was measured with the digital micrometer (Mitutoyo, Japan) with an accuracy of $0.001 \mathrm{~mm}$ at cross head speed of $1 \mathrm{~mm} / \mathrm{min} \&$ maximum load was recorded.

Figure 6 shows the mean values for Shear punch strength of three different GICs with and without coating. The highest mean value was seen with Fuji IX Extra $46.36 \pm 8.27$ followed by Fuji II LC $46.26 \pm 7.55$ and the lowest value was seen with Fuji IX Fast $24.02 \pm 3.42$ with resin coating. Without resin coating mean and standard deviation value was highest for Fuji II LC $20.05 \pm 3.40$ followed by Fuji IX Extra and lowest for Fuji IX Fast. The result obtained was statistically significant $(P<0.001)$. The interaction between GICs and Resin coating was statistically significant on Shear punch Strength in coronal sections $(F=11.929, p<0.001)$. When LSD post hoc test was applied, Fuji IX Extra, Fuji II LC mean Shear punch strength in Resin coated materials was significantly higher than uncoated materials.

In Resin coated materials, mean Shear punch strength in Fuji IX Extra and Fuji II LC was significantly higher than Fuji IX Fast. However, there was no significant difference between Fuji IX Extra and Fuji II LC. Whereas in Uncoated materials, mean Shear punch strength in Fuji II LC was significantly higher than Fuji IX Extra and Fuji IX Fast with no significant difference between Fuji IX Extra and Fuji IX Fast.

Figure 7 shows the Mean values of Surface Hardness of three different GICs with and without coating. There was significant difference on the surface hardness between the resin coated and uncoated materials. Within the cements, the highest mean value was seen with Fuji IX Extra $75.80 \pm 8.59$ followed by Fuji IX Fast $64.80 \pm 6.53$ and lowest for Fuji II LC with resin coating $64.50 \pm 5.66$. Without resin coating mean and standard value was highest for Fuji IX Extra followed by Fuji IX Fast and lowest for Fuji II LC. The results obtained were statistically significant $(P<0.01)$.

The effect of GICs on Surface hardness was statistically significant $(F=5.136, p<0.01$ ). The mean Surface hardness differ significantly between different GICs. When LSD post hoc test was applied it showed that mean Surface hardness in Fuji IXExtra was significantly higher than Fuji IX Fast and Fuji II LC. The interaction between GICs and Resin coating was statistically significant on Surface hardness ( $F=8.664, p<0.01)$. In Resin coated materials, mean Surface hardness in Fuji IX Extra was significantly higher than Fuji IX Fast and Fuji II LC with no significant difference between Fuji IX Fast and Fuji II LC. There was no significant difference between Fuji IX Extra, Fuji IX Fast and Fuji II LC for Surface hardness among the uncoated materials. 


\section{Исследования / Scientific researches}

\section{Study Design for Shear Punch Strength test}

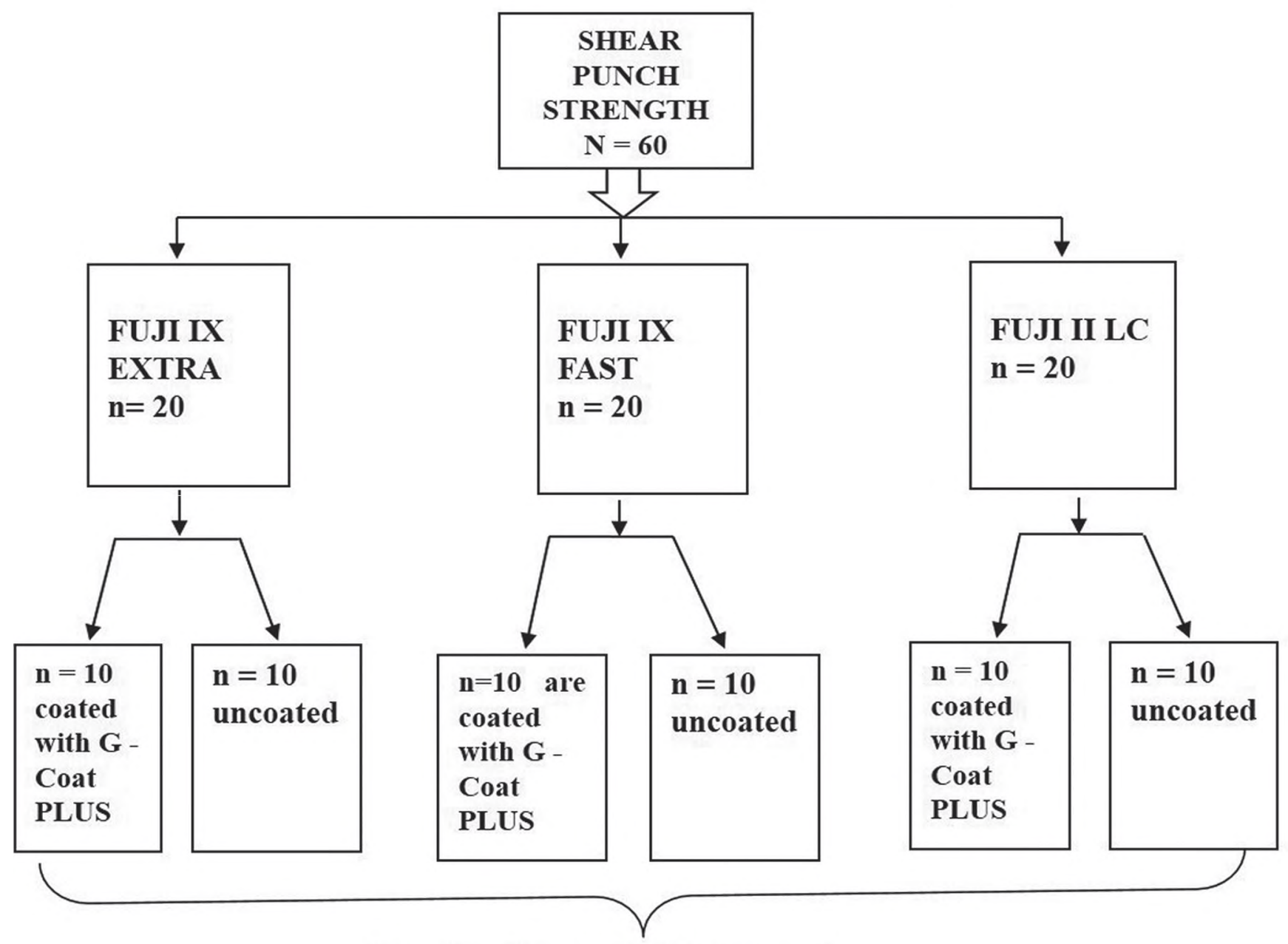

Stored in saline at $37^{0} \mathrm{C}$ for two weeks

$\downarrow$

All samples are subjected to micro punch apparatus mounted in universal testing machine.

Compressive force was applied on punch at cross head speed of $1 \mathrm{~mm} / \mathrm{min}$.

Shear punch strength was calculated using formula.

Statistical analysis and Results

Fig. 5. Schematic illustration showing protocol followed for shear punch strength test

\section{DISCUSSION}

In recentyears, therehave beenconsiderable modifications made in the formulations to improve their handling properties. In spite of these advantages, conventional glass ionomers suffer from short working times and long setting times, brittleness, low fracture toughness, poor resistance to wear, susceptible to moisture contamination or dehydration during the early stages of the setting reaction [9]. They are not the preferred restorative of choice in the posterior regions where the masticatory load is high. This makes them susceptible to fracture due to their low strength. Over the past years, several modifications have been done to enhance the physical and mechanical properties of GIC.

Surface protectors are usually recommended during their initial set inorder to protect them from moisture. Today several commercial products are available such as coca butter, petroleum jelly), waterproof varnishes (based on nitrocellulose) and resins (methyl methacrylate, amide and preferably filled, light-cured, bonding resins) varnishes $[10,11]$. Studies have shown sealing of the cement prior to initial set produces optimal compressive strength $[12,13]$. G-Coat Plus, a nano-filled self-adhesive light cured protective coating that strengthens, protects and enhances the longevity of glass ionomer restorations, composite resin and temporary restorations.

Kato et al studied the influence of the various coating materials for the conventional restorative GIC, Fuji IXGP EXTRA (F9E) on its properties and concluded that F9E coated with G-COAT PLUS showed the highest flexural strength. Tensile bond strength of G-COAT PLUS is significantly higher than other products [14]. The strength of auto-cured cements has been shown to increase over a 1-year period. A recent study found that the strength of a high strength auto-cured glass ionomer reached a maximum within 1 to 2 weeks for resin-coated and uncoated specimens, respectively [14]. 


\section{4| исследования / Scientific researches}

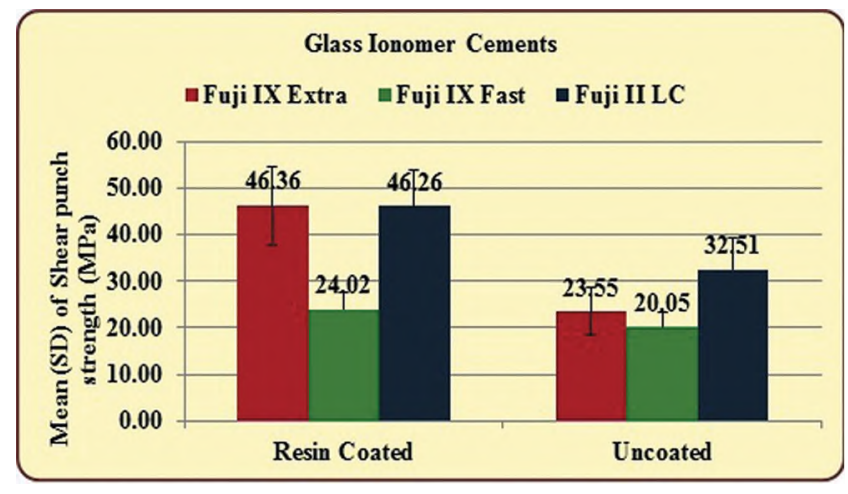

Fig. 6. Comparison of Mean and standard deviation (SD) of Shear punch strengthof three different Glass lonomer Cements (GICs) with and without resin coating

The effect of coating on two commercial glass-ionomer cements by either petroleum jelly or wax was studied by Samantha E. Booth et al [15]. Coating with wax had increased the surface hardness significantly compared with the uncoated control, whereas coating the specimens in petroleum jelly led to only a slight increase in surface hardness. This study thus confirmed the idea that immature glass-ionomers be protected from early exposure to moisture, and that the protection offered by petroleum jelly is only modest. There are studies which contradicts early contact of GIC with water, decreases the strength of GICs. The findings in our study was in agreement with Bagheri et al who determined the effect of G- Coat Plus on the mechanical properties of GICs and they concluded that the mechanical properties of the restorative materials were affected by applying G-Coat Plus and distilled water immersion over time [16].

In ourstudy, we evaluated and compared the effect of resin coating on two important properties-Surface hardness and Shear punch strength of newer modified GICs coated with a resin protective agent. The thickness of each specimen was measured with a digital micrometer with an accuracy of $0.01-\mathrm{mm}$ prior to placement in the shear punch apparatus. The mean Shear punch Strength differed significantly from the resin coated and uncoated materials. In Resin coated materials, mean Shear punch Strength in Fuji IX Extra and Fuji II LC was significantly higher than Fuji IX Fast. There was no significant difference between Fuji IX Extra and Fuji II LC. In Uncoated materials, mean Shear punch Strength in Fuji II LC was significantly higher than Fuji IX Extra and Fuji IX Fast with no significant difference between Fuji IX Extra and Fuji IX Fast. The higher strength of the resin-modified (Fuji II LC), when compared to its conventional auto-cured counterpart (Fuji II), was in agreement with previous studies [17].

Yoshaskam Agnihotri et al in 2011 evaluated the amount of micro leakage at the interface of different restorative materials and tooth surface in class II restoration. They concluded that reduction in micro leakage was seen with protective coating compared to samples without coating [18]. Within the types of restorations, Resin modified GIC showed least micro leakage followed by Polyacid modified resin composites and Resin composite.

Second important property which influences the longevity of restoration is hardness of restorative material. It is defined as the resistance to surface indentation. According to Zhang YRet al the Vickers Hardness test is a method used for brittle materials, in which a pyramidal indentation is made using a specified load and application time, the resultant hardness

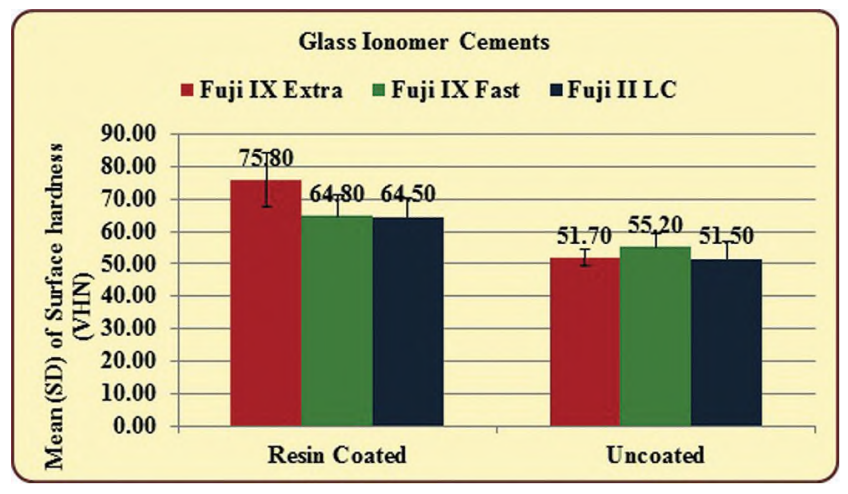

Fig. 7. Comparison of Mean and standard deviation (SD) of Surface hardness (VHN) of three different Glass lonomer Cements (GICs) with and without resin coating

number being independent of the applied load [19]. For present study the dimensions of the specimens were $10 \mathrm{~mm}$ $x 2 \mathrm{~mm}$ which is analogus to the method described by Oye bala et al [20]. The specimens coated with $\mathrm{G}$ - Coat Plus were harder than those left uncoated. The mean Surface hardness in Resin coated materials was significantly higher than uncoated materials.

D. Xie et al [21] determined the flexural strength (FS), compressive strength (CS), diametral tensile strength (DTS), Knoop hardness (KHN) and wear resistance of ten commercial glass-ionomer cements (GICs). They found that larger glass particle sizes and a more integrated microstructure contributed to a higher wear resistance. The mechanical property of GICs were closely related to their microstructures. Factors such as the integrity of the interface between the glass particles and the polymer matrix, the particle size, and the number and size of voids have important roles in determining the mechanical properties.

It is important to state that there are many factors in the oral cavity which influence the functioning and longevity of restoration i.e. micro leakage, cyclic changes of occlusal load. This present study has some limitations as it could not completely replicate the complex oral environment. The role of artificial saliva, thermo cycler was not taken into consideration. Such a lack of these conditions in vitro studies may limit the possibility of extrapolating these findings to in vivo situation.

Further in vitro and in vivo studies are recommended to correlate with the results of the present study. So, it is suggested that, in future clinical studies it is also necessary to compare the advantages of different protective agents over conventional GIC and its hybrid combinations.

\section{CONCLUSION}

The Resin coated GIC specimens showed higher shear punch strength and hardness than uncoated samples after 48 hours of kept in saline. Shear Punch Strength after G-Coat plus coating was significantly higher for Fuji IX Extra followed by Fuji II LC. Surface Hardness after GCoat plus coating was significantly higher for Fuji IX Extra followed by Fuji IXFast and Fuji II LC. Considering the above stated findings, they can be incorporated into daily clinical practice to enhance the strength and longevity of GICs.

\section{ACKNOWLEDGEMENT}

The authors would like to thank and acknowlege the assistance of Centre for Scientific Research and Development, People's College of Dental Sciences \& Research Centre, Bhopal for equipping us with the stereomicroscope. 


\section{Исследования / Scientific researches}

\section{REFERENCES:}

1. Lohbauer U. Dental GICs as Permanent Filling Materials? Properties, Limitations and Future Trends. Materials. 2010;3:76-96.

2. Wilson AD, Kent BE. Surgical cement. British Patent No. 1316129 , filed in 1969, specification published in 1973

3. Sidhu SK. Glass-ionomer cement restorative materials: a sticky subject? Aust Dent J. 2011 Jun;56 Suppl 1:23-30.

4. Sidhuyashi M, Kon M, Miyai K, Asaoka K. Strengthening of glass ionomer cement by compounding short fibres with $\mathrm{CaO}-\mathrm{P} 2 \mathrm{O} 5-\mathrm{SiO} 2$ $\mathrm{Al} 2 \mathrm{O} 3$ glass. Biom aterials. 2000;21:2051-8

5. Wang, Linda, D'alpino, Lopes PHP, Gonzaga L, Pereira JC. Mechanical properties of dental restorative materials: relative contribution of laboratory tests. J. Appl. Oral Sci. 2003:11(3):162-167.

6. Bagheri R, Tyas MJ, Burrow MF. Comparison of the effect of storage media on hardness and shear punch strength of tooth-colored restorative materials. Am J Dent. 2007;20(5):329-34

7. Saleh LA, Khaiil MF. The Effect Of Different Protective Coatings On The Surface Hardness Of GICs. The Saudi Dental Journal. 1994: 6 (1):3-7.

8. Leirskar J, Hakon Mount N, Graham J. Ngo, Hien. The influences of resin coating on the shear punch strength of a high strength autocure glass ionomer. Dental Materials. 2003;19(2):87-91.

9. Nagaraja Upadhya P, Kishore G. GIC - The Different Generations Trends Biomater. Artif. Organs 2005;18(2):158-165.

10. Williams JA, Billington RW, Pearson GJ. Effect of moisture protective coatings on the strength of a modern metal-reinforced glass-ionomer cement. J Oral Rehabil. 1998;25(7):535-40

11. Hattab FN, Amin WM. Fluoride release from glass ionomer restorative materials and the effects of surface coating. Biomaterials 2001;22:1449-1458

\section{AUTHOR INFORMATION:}

Dr. Niharika Mishra ${ }^{1}$ - MDS, Assistant professor, Department of Conservative Dentistry \& Endodontics Mansarovar Dental College Hospital and Research Center, Bhopal, ORCID ID: 0000-0002-1065-4871.

Dr. Manish AgarwaF - MDS, HOD, Department of Conservative Dentistry \& Endodontics People's College of Dental Sciences and Research Centre, Bhopal.

Dr. MP Singh ${ }^{3}$ - MDS, Professor and HOD, Dentistry Department, Chirayu Medical College and Hospital, Bhopal

Dr. Apoona Khullar - MDS, Senior Lecturer, Rishiraj College of Dental Science and Research Centre, Bhopal

Dr. Khushboo Magwa ${ }^{2}$ - MDS, Assistant Professor, Department of Conservative Dentistry \& Endodontics People's

College of Dental Sciences and Research Centre, Bhopal.

Dr. Sonal Singh Thakur ${ }^{5}$ - MDS, Senior Lecturer, Deparment of Conservative Dentistry \& Endodontics Sri Aurobindo College of Dentistry, Indore.

${ }^{1}$ Department of Conservative Dentistry \& Endodontics, Mansarovar Dental College Hospital and Research Centre, Bhopal, India.

²Department of Conservative Dentistry \& Endodontics, People's College of Dental Sciences and Research Centre, Bhopal, India.

${ }^{3}$ Dentistry Department, Chirayu Medical College and Hospital, Bhopal, India.

${ }^{4}$ Department of Conservative Dentistry \& Endodontics, Rishiraj College of Dental Science and Research Centre, Bhopal India.

${ }^{5}$ Department of Conservative Dentistry \& Endodontics, Sri Aurobindo college of dentistry, Indore, India.

Coordinates for communication with authors: 